\title{
Die zahnärztliche selektive Intensivprophylaxe in Rheinland-Pfalz: Untersuchungen an Erstklässlern im Schuljahr 2013/2014
}

\author{
The Intensified Dental Preventive Programme in Rhineland-Palati- \\ nate: Investigations on first-grade school-children in 2013/2014
}

\section{(ㄷ) (i) () $\ominus$}

\section{Autoren}

J. Weusmann ${ }^{1}$, B. Mahmoodi' ${ }^{1}$ K. Kordsmeyer ${ }^{1}$, A. Azaripour ${ }^{1}$, C. Walter ${ }^{2}$, B. Willershausen ${ }^{1}$

\section{Institute}

1 Poliklinik für Zahnerhaltungskunde und Parodontologie, Universitätsmedizin der Johannes-Gutenberg-Universität Mainz, Mainz

2 Klinik und Poliklinik für Mund-, Kiefer- und Gesichtschirurgie - Plastische Operationen-, Universitätsmedizin Mainz, Mainz

\section{Schlüsselwörter}

Karies, Kindheit, SIP, Mundgesundheit, Selektive Intensivprophylaxe

\section{Key words}

caries, childhood, SIP, oral health, intensified dental preventive programme

\section{Bibliografie}

DOI http://dx.doi.org/10.1055/s-0042-108582

Online-Publikation: 14.6.2016 | Gesundheitswesen 2017; 79: 247-251

(c) Georg Thieme Verlag KG Stuttgart · New York

ISSN 0941-3790

Korrespondenzadresse

Dr. med. dent. Jens Weusmann

Poliklinik für Zahnerhaltungskunde und Parodontologie

Universitätsmedizin der Johannes-Gutenberg-Universität Mainz

Augustusplatz 2

55131 Mainz

jens.weusmann@unimedizin-mainz.de

\section{ZUSAMMENFASSUNG}

Ziel der Studie Für Kinder und Jugendliche mit stark erhöhtem Kariesrisiko wurde durch die LAGZ (Landesarbeitsgemeinschaft Jugendzahnpflege) Rheinland-Pfalz im Jahr 2004 die selektive Intensivprophylaxe (SIP) etabliert. Vergleichbare Programme existieren in den meisten deutschen Bundesländern in unterschiedlicher Ausprägung. Die Ergebnisse der schulzahnärztlichen Untersuchungen der Erstklässler im Bundesland Rheinland-Pfalz im Schuljahr 2013/2014 werden nach Prophylaxeprogramm („Aktivprogramm Jugendzahnpflege“ [SIP] gegenüber Standardprogramm [SP]) betrachtet. Hierbei sollen die innerschuljährlichen Auswirkungen auf das teilnehmende Kollektiv beleuchtet werden. Des Weiteren soll untersucht werden, ob die SIP tatsächlich die Kinder mit der höchsten Karieserfahrung erreicht.

Methodik Instruierte Schulzahnärzte in Rheinland-Pfalz erhoben im Schuljahr 2013/2014 den d3mft/D3MFT-Index nach WHO-Kriterien. Gemäß diesen wurde Karies bei Dentinbefall als solche diagnostiziert; röntgenologische Methoden oder fiberoptische Methoden standen nicht zur Verfügung. Die Untersuchungen fanden schuljährlich einmalig im SP; 2-malig in der SIP statt. Schüler und Eltern wurden bei Behand- lungsbedarf stets informiert und nach daraufhin nicht erfolgter Vorstellung beim Zahnarzt einmalig erinnert. Aus den erhobenen $\mathrm{d} 3 \mathrm{mft} /$ D3MFT-Werten wurde der Significant Caries Index (SiC) berechnet. Die statistischen Berechnungen wurden mit SPSS 22.00. durchgeführt. Ergebnisse Insgesamt 25020 Erstklässler waren nach d3mft/D3MFT auswertbar. Hiervon nahmen $\mathrm{n}=1164$ Erstklässler im Schuljahr 2013/2014 am SIP in Rheinland-Pfalz teil; bei $n=1002$ lagen die Ergebnisse beider zahnärztlichen Untersuchungen vor. Die Karieserfahrung der Erstklässler in der SIP überstieg signifikant diejenige der Teilnehmer des SP (41,8\% Naturgesunde gegenüber 61,7\%, p<0,0001), d3mft 2,71 gegenüber 1,21, $p<0,0001)$. Deutlich erkennbar war der Unterschied zwischen 1 . Untersuchung und 2. Untersuchung in der SIP besonders hinsichtlich des zahnbezogenen ( $43,2 \%$ gegenüber $54,2 \%, p<0,0001)$ und individuellen Sanierungsgrades ( $16,0 \%$ gegenüber $23,0 \%, p<0,0001$ ). Schlussfolgerung Die Ergebnisse der vorliegenden Studie zeigen, dass die SIP in Rheinland-Pfalz tatsächlich in einer besonders karieserfahrenen Population durchgeführt wird. Der signifikante Unterschied bezüglich Sanierungsgrad zwischen 1. und 2. Untersuchung lässt den Schluss zu, dass häufig Aufgrund von schulzahnärztlich diagnostiziertem Behandlungsbedarf ein Zahnarzt zur Therapie aufgesucht wurde.

\section{ABSTRACT}

Aim For children and adolescents at increased risk of caries, the "Selective Intensive Prophylaxis" (SIP) programme was established in RhinelandPalatinate in 2004, as well as in the most other German states. This study compares the results of the school dentists' evaluations of first grade school children in 2013/2014 participating in the "Selective Intensive Prophylaxis" (SIP) programme or the "Standard Programme" (SP).

Methods The d3mft/D3MFT index was recorded by school dentists according to the WHO criteria; caries was diagnosed if dentin was affected; no radiography or fiber-optic transillumination was used. The examinations were performed once under the SP and twice under the SIP. Children and parents were informed in case of a need for treatment and reminded if the did not see the family dentist. Out of the evaluated d3mft/D3MFT values, the Significant Caries Index ( $\mathrm{SiC}$ ) was calculated. Statistical computing was performed using SPSS 22.00.

Results Of all first graders, $\mathrm{n}=25020$ were evaluable for $\mathrm{d} 3 \mathrm{mft} / \mathrm{D} 3 \mathrm{MFT}$. Altogether $n=1164$ first graders were included into the SIP in the 2013/2014 term; for $n=1002$ of those, the results for both examinations were available. The caries experience of those pupils participating in the SIP was clearly higher than in the SP ( $41.8 \%$ naturally healthy vs. $61.7 \%, p<0.0001)$. There was significant difference between the first and second examination in the SIP, especially with respect to individual ( 16.0 vs. $23.0 \%, p<0.0001)$ and tooth-related (43.2 vs. $54.2 \%$, $\mathrm{p}<0.0001)$ level of restoration.

Conclusion The results show that the SIP in Rhineland-Palatinate is conducted in a population with high caries experience. The significant difference concerning the dental restoration level, both individual- and teethrelated - leads to the conclusion that children frequently sought dental treatment if a need for treatment was diagnosed by the school dentist. 


\section{Einleitung}

Nach wie vor ist Karies als multifaktorielle Erkrankung der Zahnhartgewebe durch Mikroorganismen des Biofilms eine der häufigsten Krankheiten im Kindesalter. So tritt sie bei 5- bis 17-jährigen US-Amerikanern mehr als 5-mal so häufig auf wie Asthma und mehr als siebenmal so häufig wie Heuschnupfen [1].

Im Jahr 2003 formulierten die World Health Organization (WHO), Fédération Dentaire Internationale (FDI) und International Association for Dental Research (IADR) die Forderung, die spezifischen Mundgesundheitsziele unter Einbeziehung des politischen, sozioökonomischen und gesetzgeberischen Zusammenhangs neu zu setzen [2]. Die Entscheidung bezüglich der konkreten Ziele sollte der jeweiligen Zuständigkeit überlassen werden. Dem folgend gab die Deutsche Zahnärztekammer das Ziel vor, dass 80 \% der Sechsjährigen im Jahr 2020 eine kariesfreie erste Dentition aufweisen sollen [3].

Eine ausgeprägte Karieserfahrung in frühen Lebensjahren ist prädiktiv für ein erhöhtes Kariesrisiko im Erwachsenenalter [4]. Deshalb spielt Kariesprävention ab früher Kindheit eine wichtige Rolle, zumal ausgeprägte Karies die Fähigkeit zu schlafen, zu essen und z. B. auch Schulaufgaben zu erledigen, negativ beeinflussen kann [5].

Vielfältige präventive Maßnahmen wurden vorgenommen; von topischer und systemischer Fluoridierung über Fissurenversiegelung bis hin zu neuen Ansätzen wie oralen Probiotika [6]. Hiervon erscheint die Fluoridlackapplikation als jahrzehntelang bewährte Maßnahme besonders nützlich [7].

In Deutschland wird die „Verhütung von Zahnerkrankungen (Gruppenprophylaxe)“ durch das SGB V § 21 geregelt. Hier heißt es in Abs. 1: „Für Kinder mit besonders hohem Kariesrisiko sind spezifische Programme zu entwickeln.“

Auf nationaler Ebene wird die Prävention von der Deutschen Arbeitsgemeinschaft für Jugenzahnpflege (DAJ) koordiniert, darüber hinaus arbeiten weitere Organisationen auf Landes- und Kreisebene mit Schulen und Kindergärten zusammen.

Den gesetzlichen Anforderungen folgend hat in Rheinland-Pfalz die Landesarbeitsgemeinschaft für Jugendzahnpflege (LAGZ) das „Aktivprogramm Zahnvorsorge“ aufgelegt, um die Präventionsmaßnahmen für jene in besonderem Maße karieserfahrenen Kinder zu intensivieren.

An diesem Aktivprogramm (SIP) nahmen ca. 80 von insgesamt 970 Schulen mit Erstklässlern in Rheinland-Pfalz teil. Die Auswahl wird von der LAGZ und den zuständigen Ministerien anhand des Kariesrisikoprofils der Schulen basierend auf den regelmäßig durchgeführten epidemiologischen Begleituntersuchungen und freiwilligen Bewerbungen der Schulen getroffen [8]. Die SIP in RheinlandPfalz beinhaltet 2 Untersuchungen des Schulzahnarztes pro Schuljahr und umfasst auch die Erfolgskontrolle durch Reihenuntersuchungen. An den Nicht-Hochprävalenz-Schulen werden eine Schulstunde Prophylaxegespräch sowie eine Untersuchung durchgeführt. Im Aktivprogramm wird bei einem ersten Besuch des Schulzahnarztes nach Schuljahresbeginn (August - Oktober) ein Prophylaxegespräch mit Erläuterung der anschließenden ersten Untersuchung mit Zahnputzübung über insgesamt 2 Schulstunden pro Klasse absolviert.

Beim zweiten Besuch (Januar - Februar) werden Mundhygieneübung, Prophylaxegespräch und eine erste Fluoridierung mit wie- derum 2 Schulstunden pro Klasse veranschlagt. Eine Untersuchung findet zu diesem Zeitpunkt jedoch nicht statt.

Beim dritten Besuch zwischen Ostern und bis Juni erfolgt eine zweite Untersuchung, mit erneuter Fluoridierung, Prophylaxegespräch und Zahnputzübung ebenfalls für 2 Schulstunden pro Klasse [8].

Die Ergebnisse des Gesamtkollektivs der Erstklässler im Schuljahr 2013/2014 bestätigten den anhaltenden Trend zu verbesserter Zahngesundheit bei Erstklässlern. Hier war von 25020 Kindern der d3mft auswertbar. Im Durchschnitt lag dieser Wert bei $1,28 \pm 2,27$ bei einem SiC-Wert von 3,73 $\pm 2,51$ [9].

Ziel der vorliegenden Untersuchung war es, die kurzfristigen Effekte des Aktivprogramms Jugendzahnpflege (SIP) in RheinlandPfalz zu überprüfen, sowie die Zahngesundheit der Teilnehmer mit denjenigen Erstklässlern zu vergleichen, die am Standard-Prophylaxeprogramm (SP) teilgenommen haben. Hierbei ist von Interesse, ob die SIP tatsächlich die Kinder mit der größten Karieserfahrung erreichen kann.

\section{Methodik}

Die Untersuchungen wurden in den Räumlichkeiten der jeweiligen besuchten Schulen durchgeführt. Die beteiligten Zahnärzte wurden mittels einer Broschüre bezüglich der Karieskriterien instruiert, letztere basierten auf den Kriterien der WHO, die die Karies in 4 Stadien einteilt. Röntgenologische oder fiberoptische Verfahren fanden keine Anwendung, sondiert wurde lediglich in Ausnahmefällen.

Der dmft/DMFT-Wert ist seit über 75 Jahren in Gebrauch und bleibt der am häufigsten verwendete Index zur Kariesbestimmung weltweit [10]. Im Rahmen der Untersuchungen wurde der d3mft/ D3MFT-Wert der Erstklässler bestimmt. Der Buchstabe d/D bezeichnet einen kariös zerstörten Zahn (decayed), m/M einen kariös bedingt fehlenden Zahn (missing) und f/F einen gefüllten Zahn (filled). Während die Großbuchstaben für das bleibende Gebiss stehen, finden die kleinen Buchstaben für das Milchgebiss Verwendung. Die Ziffer „3“ besagt, dass Karies als solche diagnostiziert wurde, wenn bereits Dentinbefall vorlag.

Um den Kariesbefall von Hochrisikopopulationen besser erfassen zu können, wurde aus dem d3mft der „Significant Caries Index“ (SiC) nach Bratthall bestimmt. Er beschreibt den mittleren d3mft desjenigen Drittels der Population, das die höchste Kariesaktivität aufweist [11].

Zusätzlich zur Bestimmung der d3mft/D3MFT-Werte wurden die Kinder durch den untersuchenden Zahnarzt in verschiedene Kategorien eingeteilt: „Naturgesund“ (wenn d3mft+D3MFT=0); "Saniert/Kein Behandlungsbedarf “ (wenn $F(T) / f(t)>0$ bei gleichzeitigem d3mt/D3MT =0); Behandlungsbedarf, wenn d3t/ D3T >0 . „Erhöhtes Kariesrisiko“ wurde diagnostiziert wenn $\mathrm{d} 3 \mathrm{mft}+\mathrm{D} 3 \mathrm{MFT}>5$ und/oder D3(T) $>0$. Die Eltern wurden vor der stattfindenden Untersuchung schriftlich informiert. Unmittelbar nach der Untersuchung wurden sie per Brief über die Untersuchungsergebnisse ihres Kindes in Kenntnis gesetzt sowie, falls notwendig, ein Zahnarztbesuch zu Therapiezwecken empfohlen.

Der zweiseitige Mann-Whitney U-Test wurde durchgeführt, um die Indizes zu vergleichen, während zum Vergleich von Individuenzahlen Chi-Quadrat-Tests durchgeführt wurden. IBM SPSS 22.00. 
wurde zur Datenanalyse verwendet. Das Signifikanzniveau lag bei $5 \%$. Werte werden als Mittelwerte \pm der Standardabweichung angegeben.

Die vorliegenden Daten wurden durch die Landesarbeitsgemeinschaft Jugendzahnpflege (LAGZ) Rheinland-Pfalz erhoben und wurden anonymisiert. Auf Grundlage der genannten Daten wurde bereits ein Artikel veröffentlicht, der die Ergebnisse des Gesamtkollektivs thematisiert [9].

Die Ethikkommission wurde von der Studie in Kenntnis gesetzt. Auf Basis des Vorliegens einer retrospektiven Analyse anonymisierter Daten sei ein Ethikvotum nicht notwendig (Schreiben der Ethikkommission vom 13.05.2015).

\section{Ergebnisse}

Im Schuljahr 2013/14 nahmen von insgesamt $n=32640$ in Rheinland-Pfalz eingeschulten Erstklässlern $n=1164$ Schülerinnen und Schüler am ersten Untersuchungstermin im „Aktivprogramm Zahnvorsorge“ (SIP) teil. Hiervon waren 46,7\% weiblichen Geschlechts.

Von allen o.g. SIP-Teilnehmern nahmen $86,1 \%(n=1002)$ den zweiten Untersuchungstermin wahr. Die übrigen 13,9\% wurden u. a. wegen Abwesenheit, bedingt durch Schul- bzw. Klassenstufenwechsel kein zweites Mal erfasst.

Als Vergleichsgröße wurden die Ergebnisse der Untersuchungen des Gesamtkollektivs sowie des SPs im Land Rheinland-Pfalz im selben Schuljahr herangezogen [9].

- Abb. 1,2 sowie $>$ Tab. $\mathbf{1}$ zeigen die Ergebnisse der Untersuchungen sowie der Teilnehmer der SIP neben denjenigen des SP.

Hier ergaben sich im Vergleich der beiden Kollektive erwartungsgemäß Unterschiede, so ist der Anteil naturgesunder Kinder ist in der SIP-Gruppe um annähernd $20 \%$ niedriger (61,7\% gegenüber $41,8 \%)(p<0,0001)$.

Des Weiteren fiel der d3mft-Wert in der SIP-Gruppe mit $2,71 \pm 3,23$ (1. Untersuchung) bzw. 2,86 $\pm 3,23$ (2. Untersuchung) $(p=0,121)$ deutlich höher aus als in der SP-Gruppe mit 1,21 $\pm 2,18$ $(p<0,0001)$. Einen d3mft-Wert von 0 wiesen 63,6\% der Kinder des SP, jedoch nur 43,2\% bei der 1. bzw. 40,0\% in der SIP bei der 2 . Untersuchung auf ( $\triangleright$ Abb. 2). Ein d3mft-Wert von 10 oder höher war bei den SIP-Kindern mit 3,0\% fast 4-mal so häufig wie beim SP mit $0,8 \%$. Der in der SIP erwartungsgemäß große Anteil an Kindern mit hohen d3mft-Werten spiegelt sich auch im SiC wider, mit einem Wert von 6,74 $\pm 2,14$ (1. Untersuchung) bzw. 6,85 $\pm 2,09$ (2. Untersuchung) in der SIP vs. einem SiC 3,55 2,45 im SP ( $<<0,0001)$.

Beim Vergleich der Befunde zum Zeitpunkt des 1. und des 2. Untersuchungstermins bei Teilnehmern der SIP fällt auf, dass der Anteil an naturgesunden Gebissen bei der zweiten Untersuchung um $4,5 \%$ von $41,8 \%$ auf $37,3 \%$ zurückgegangen ist ( $p=0,028)$. Gleichzeitig stieg der Anteil der Kinder mit vollständiger Zahnsanierung um 7 von 16,0\% beim ersten auf $23,0 \%$ beim zweiten Besuch hochsignifikant an $(p<0,0001)$. Der Anteil der behandlungsbedürftigen Kinder sank nicht signifikant von 41,7 auf 39,3\% $(p=0,353)$; genauso wie der Prozentsatz der Kinder mit „erhöhtem Kariesrisiko" von 25,1 auf 22,3\% ( $=0,3)$ (

Zwischen den $\mathrm{d} 3 \mathrm{mft}$-Werten von Jungen und Mädchen ergaben sich weder bei der 1. $(p=0,29)$ noch bei der 2 . Untersuchung $(p=0,19)$ signifikante Unterschiede.

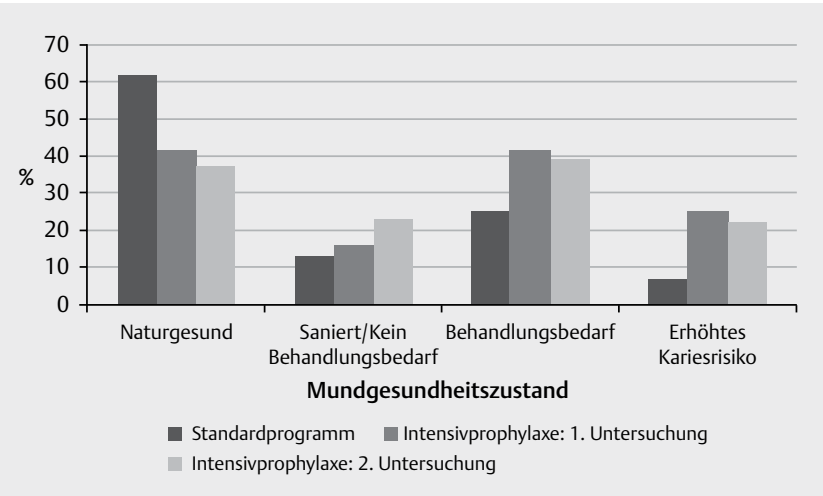

Abb. 1 Longitudinaler Verlauf der Zahngesundheit, des Kariesrisikos und des Behandlungsbedarfs der an der SIP teilnehmenden Kinder der 1. Klasse im Vergleich zu Kindern im SP in Rheinland-Pfalz im Schuljahr 2013/14.

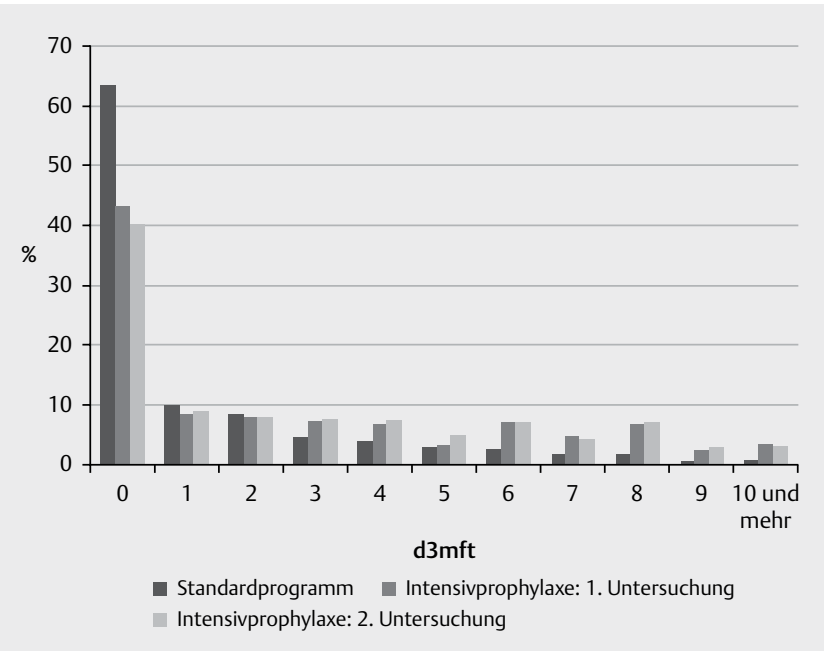

Abb. 2 d3mft-Verteilung der Kinder der 1. Klasse in RheinlandPfalz nach Prophylaxeprogramm im Schuljahr 2013/14.

Im Hinblick auf den zahnbezogenen Sanierungsgrad zeigt sich ein hochsignifikanter Anstieg ( $p<0,0001)$ zwischen 1. und 2. Untersuchung. Demgegenüber sank der Anteil der als „decayed“ befundeten Zähne $(p=0,02)(\triangleright$ Tab. 1).

\section{Diskussion}

Mit Blick auf die Untersuchungsergebnisse fällt der große Unterschied in der Karieserfahrung zwischen SIP und SP auf, welcher angesichts der häufig beschriebenen interindividuellen Kariespolarisierung $[12,13]$ auch zu erwarten war. In Anbetracht der Tatsache, dass das am meisten karieserfahrene Drittel des SP $(n=7952)$ einen höheren d3mft-Wert aufweist als das Gesamtkollektiv ( $n=1164)$ der SIP (3,55 gegenüber 2,71) wird deutlich, dass durch die SIP zwar karieserfahrene Kinder erreicht werden, jedoch noch nicht so flächendeckend, wie dies wünschenswert wäre. Deshalb sollte ein Ziel sein, möglichst noch mehr von Karies betroffenen Kindern noch früher intensivierte Prophylaxemaßnahmen angedeihen zu lassen. 
- Tab. 1 d3mft/D3MFT-Mittelwerte und Significant Caries Index (SiC) der Erstklässler in Rheinland-Pfalz im Prophylaxeprogramm des Schuljahrs 2013/14.

\begin{tabular}{|l|c|c|c|c|c|c|c|}
\hline & d3mft & d3t & mt & $\mathbf{f t}$ & D3MFT & SiC (d3mft) & Sanierungsgrad (mt+ ft)/d3mft \\
\hline SP & 1,21 & 0,67 & 0,16 & 0,39 & 0,04 & 3,55 & $45,5 \%$ \\
\hline SIP: 1. U. & 2,71 & 1,54 & 0,30 & 0,87 & 0,09 & 6,74 & $43,2 \%$ \\
\hline SIP: 2. U. & 2,86 & 1,31 & 0,43 & 1,12 & 0,14 & 6,85 & $54,2 \%$ \\
\hline
\end{tabular}

Vergleicht man die Ergebnisse dieser Studie mit denen des Gesamtkollektivs in Rheinland-Pfalz, zeigt sich, dass durch die relativ wenigen Kinder der SIP ein d3mft-Anstieg um 0,07 verursacht wird (1,21 im SP gegenüber 1,28 im Gesamtkollektiv) [9]. Dieser Wert unterstreicht erneut die nach wie vor existente Kariespolarisierung in der SIP.

Die langfristige Wirksamkeit einer intensivierten Gruppenprophylaxe (SIP) konnte von Pieper et al. nachgewiesen werden. Waren die d3-6mft/D3-6MFT-Werte in der Gruppe, in der die „selektive Intensivprophylaxe“ betrieben wurde, in der ersten Klasse annähernd derjenigen der Vergleichspopulation, wenn auch die dmft/ DMFT-Werte in beiden Populationen höher waren als in der SIPGruppe in unserer Studie $(3,12$ bzw. 3,28), so war die Überlegenheit im longitudinalen Vergleich nach 6 Jahren jedoch hochsignifikant mit einem d3-6mft/D3-6MFT Score von 0,88 in der SIP-Gruppe gegenüber 1,72 in der Kontrollpopulation ( $p<0,0005)$ [14]. Die kleineren Scores zum zweiten Untersuchungszeitpunkt erklären sich durch den Verlust entsprechender kariöser bzw. gefüllter Zähne bzw. die Substitution durch einen gesunden Zahn der zweiten Dentition.

Bei Betrachtung der Unterschiede zwischen 1. und 2. Untersuchungstermin - einem wesentlich kürzeren Zeitraum - in unserer Studie zeigt sich, dass sich der d3mft-Wert nicht signifikant erhöht hat. Der hochsignifikante Anstieg des individuellen Sanierungsgrades um $7 \%$ ist durchaus positiv zu bewerten; der signifikante Anstieg des zahnbezogenen Sanierungsgrades untermauert dies. Die Tatsache, dass der Anteil der Kinder mit Behandlungsbedarf bei weitem nicht im selben Maße absank, lässt sich durch neu entstandene Läsionen erklären. Hier zeigt sich einerseits zwar der leichte Anstieg der Kariesprävalenz innerhalb eines Schuljahres, andererseits jedoch auch die Wirksamkeit von Befundung und Therapieempfehlung, gerade beim Blick auf den Sanierungsgrad.

Dennoch ist der Fakt, dass sich dieser Anteil der behandlungsbedürftigen Kinder - genauso wie der Anteil der Kinder, der die festgelegten Kriterien für „erhöhtes Kariesrisiko“ erfüllt - kaum ändert, kritisch zu betrachten und stellt die Frage nach Steigerungspotenzialen in der Effektivität. Da es letztenendes den Eltern obliegt, ob sie eine etwaig ausgesprochene Therapieempfehlung durch den Schulzahnarzt für ihr Kind auch tatsächlich wahrnehmen und Evidenz für den Einfluss des elterlichen Mundhygieneverhaltens auf die kindliche Mundgesundheit besteht [15], wäre anzudenken, verstärkt bei der Elternaufklärung anzusetzen.

Davon abgesehen ist die Mehrzahl der an der SIP teilnehmenden Schulen Förderschulen, nicht selten ist hier die kindliche Mundhygiene durch motorische und geistige Einschränkungen beeinträchtigt [16].

Limitierende Faktoren in der vorliegenden Studie sind die hohe Anzahl unterschiedlicher Untersucher $(n=77)$ sowie die Tatsache, dass Karies als solche erst bei Dentinbefall diagnostiziert wurde.
Letzteres erfolgte, um trotz der Tatsache, dass mehrere Untersucher tätig waren, nur wirklich gesicherte Kariesdiagnosen als solche zuzulassen, was wiederum zu Lasten der Sensitivität gegangen sein mag. Darüber hinaus wurde die Untersuchung üblicherweise in der Schule ohne Luftpuster und zahnärztlichen Stuhl durchgeführt.

Da in den letzten Jahrzehnten zahnfarbene Restaurationen in Lehre und Praxis auf dem Vormarsch sind, ist es denkbar, dass gefüllte Zähne nicht als solche diagnostiziert wurden [17].

Ob die Schulkinder des SP mit kariösen Befunden in ähnlichem Maße von Befundung und Therapieempfehlung profitieren wie diejenigen in der SIP, lässt sich leider nicht nachvollziehen. Zur Verifizierung wäre auch im SP eine Zweituntersuchung von Interesse, die jedoch nicht standardmäßig durchgeführt wird.

Wie es Präventionsmaßnahmen immanent ist, dürfte der Effekt der simultan zu den Untersuchungen durchgeführten Fluoridierungsmaßnahmen sowie derjenige des Prophylaxeunterrichts eher langfristiger Natur sein. Die weiterhin ungebrochene Tendenz zum Rückgang der Kariesprävalenz im Gesamtkollektiv ist möglicherweise einerseits dem steigenden Marktanteil des fluoridierten Speisesalzes zuzuschreiben, andererseits mag die Etablierung systematische Gruppenprophylaxe eine Rolle gespielt haben [18, 19]. Es ist weiterhin denkbar, dass die heutige Erstklässlergeneration vom Gruppenprophylaxeunterricht ihrer Eltern zu Schulzeiten profitiert.

Zahlreiche Studien zeigen einen Zusammenhang zwischen dem sozioökonomischen Status des Kindes und der Kariesneigung $[4,20]$. Es wird eine große Herausforderung sein, möglichst alle Kinder profitieren zu lassen, die am meisten Karieserfahrung aufweisen. Möglicherweise müsste hierzu bereits im Vorschulalter angesetzt werden, gegebenenfalls durch Reihenuntersuchungen mit Verweisungssystem im Kindergarten.

Zusammenfassend lässt sich feststellen: Die Zahngesundheit der Schülerinnen und Schüler verbessert sich durch die selektive Intensivprophylaxe bereits kurzfristig innerhalb eines Schuljahres; viele an der SIP teilnehmende Erstklässler profitieren unmittelbar von der zahnärztlichen Befunderhebung und der angeschlossenen Therapieempfehlung im Bedarfsfall. Spannend wäre es auch hier, Ergebnisse longitudinaler Beobachtungen über einen größeren Zeitraum sichten zu können. Des Weiteren sollte versucht werden, Hochrisikopopulationen noch früher und effektiver zu identifizieren um dort gezielte Aufklärungsarbeit zu leisten, d. h. man könnte sich analoge Programme ggf. bereits im Kindergarten und unter stärkerer Einbeziehung der Eltern vorstellen.

\section{Interessenkonflikt}

Die Autoren geben an, dass kein Interessenkonflikt besteht. 


\section{Literatur}

[1] $\mathrm{NIH}($ USA). US Department of Health and Human Services: Oral Health in America: A Report of the Surgeon General. In: Rockville MD. USA: US Department of Health and Human Services, National Institute of Dental and Craniofacial Research, National Institutes of Health; 2000

[2] Hobdell M, Petersen PE, Clarkson J et al. Global goals for oral health 2020. International dental journal 2003; 53: 285-288

[3] Ziller S, Micheelis W, Oesterreich D et al. Goals for oral health in Germany 2020. International dental journal 2006; 56: 29-32

[4] Thomson WM, Poulton R, Milne B] et al. Socioeconomic inequalities in oral health in childhood and adulthood in a birth cohort. Community dentistry and oral epidemiology 2004; 32: 345-353

[5] [Anonym]. Oral health: prevention is key. Lancet. 2009; 373: 1

[6] Twetman S, Stecksen-Blicks C. Probiotics and oral health effects in children. International journal of paediatric dentistry / the British Paedodontic Society [and] the International Association of Dentistry for Children 2008; 18: 3-10

[7] Kay E, Locker D. A systematic review of the effectiveness of health promotion aimed at improving oral health. Community dental health 1998; 15: 132-144

[8] Landesarbeitsgemeinschaft Jugendzahnpflege Rheinland-Pfalz. Aktivprogramm Zahnvorsorge. In

[9] Weusmann J, Mahmoodi B, Azaripour A et al. Epidemiologica investigation of caries prevalence in first grade school children in Rhineland-Palatinate, Germany. Head \& face medicine 2015; 11: 33

[10] Broadbent JM, Thomson WM. For debate: problems with the DMF index pertinent to dental caries data analysis. Community dentistry and oral epidemiology 2005; 33: 400-409

[11] Bratthall D. Introducing the Significant Caries Index together with a proposal for a new global oral health goal for 12 -year-olds. International dental journal 2000; 50: 378-384
[12] [Anonym]. Vierte Deutsche Mundgesundheitsstudie - (DMS IV) Neue Ergebnisse zu oralen Erkrankungsprävalenzen, Risikogruppen und zum zahnärztlichen Versorgungsgrad in Deutschland. 2005; Dt. Ärzte-Verl.; 2006

[13] Hellwig E, Altenburger M. Paradigm shift in conservative dentistry: from a mechanistic to a prevention-oriented perception. Bundesgesundheitsblatt, Gesundheitsforschung, Gesundheitsschutz 2011; 54: 1015-1021

[14] Pieper K, Weber K, Stein S et al. Evaluation of an Intensified Dental Preventive Programme Aimed at Children with Increased Caries Risk. Gesundheitswesen (Bundesverband der Arzte des Offentlichen Gesundheitsdienstes (Germany)) 2013, doi:10.1055/s-0032-1333245

[15] Castilho AR, Mialhe FL, Barbosa Tde $S$ et al. Influence of family environment on children's oral health: a systematic review. Jornal de pediatria 2013; 89: 116-123

[16] Altun C, Guven G, Akgun OM et al. Oral health status of disabled individuals attending special schools. European journal of dentistry 2010; 4: 361-366

[17] Bucher K, Metz I, Pitchika V et al. Survival characteristics of composite restorations in primary teeth. Clinical oral investigations 2014, doi:10.1007/s00784-014-1389-9

[18] Pieper K. Epidemiologische Begleituntersuchung zur Gruppenprophylaxe. 2009 In: e.V.) DDAfj ed. Bonn; 2010

[19] Marthaler TM. Salt fluoridation and oral health. Acta medica academica 2013; 42: 140-155

[20] Schwendicke F, Dorfer CE, Schlattmann P et al. Socioeconomic inequality and caries: a systematic review and meta-analysis. Journal of dental research 2015; 94: 10-18 